\title{
Human cardiac wall stress analysis with patient-specific myocardial material properties
}

\author{
A. Chien ${ }^{1}$, R. M. Shoucri ${ }^{2}$, A. $\mathrm{Mal}^{3}$ \& C. Montemagno ${ }^{1}$ \\ ${ }^{I}$ Department of Bioengineering, University of California, Los Angeles, \\ USA \\ ${ }^{2}$ Department of Mathematics and Computer Science, \\ Royal Military College of Canada, Kingston, Ontario, Canada \\ ${ }^{3}$ Department of Mechanical and Aerospace Engineering, \\ University of California, Los Angeles, USA
}

\begin{abstract}
A patient-specific model to evaluate cardiac function from a beating heart is needed. Most current cardiac models were constructed using post mortem measurements of animal hearts. To help understand human cardiac mechanics and disease development, a model constructed based on real patient data is important. In this study, we have employed medical imaging techniques to analyze myocardial wall stress. We developed a methodology to determine myocardial material properties using measurements from magnetic resonance images. The finite element method was used to combine this information and perform wall stress calculations. This process was applied to two healthy human subjects to compute myocardial wall stress. We found general agreement in myocardial wall stress between subjects, with results showing higher stress at the inner wall of ventricles. The wall stress distributions in the left ventricle differed between the two subjects; one had a large area of high wall stress, the other had discontinuous bands of higher wall stress.
\end{abstract}

Keywords: cardiac mechanics, myocardial wall stress, patient-specific modelling, myocardial material properties, and finite element analysis.

\section{Introduction}

To gain insight into ventricular dysfunction a quantitative method to describe myocardial mechanics in a beating heart has been intensively sought. Research 
to understand ventricular function including approaches from various fields, such as finite element computation, strain measurement, systematic simulation, and electrophysiology [1-3]. Among these, finite element (FE) computation based on mechanical deformation theory provides the potential to quantify gross myocardial function. By calculating myocardial wall stress (WS) it can be used to quantify myocardial function and oxygen consumption [4]. Its potential to direct surgical planning and therapeutic development is considerable $[5,6]$.

FE computation in ventricular function has been utilized to understand the local cardiac elasticity and ventricular geometry remodelling process [7-9]. The key elements for FE computation are the particular mechanical deformation theory and the material properties of the myocardium. These material properties have typically been derived from post mortem measurements of animal myocardial tissue $[1,7,8]$. In vivo studies in cardiac mechanics are extremely rare. Recently, with advancements in medical imaging techniques, more accurate cardiac motion has been revealed, suggesting that myocardial material properties are different among individuals and may play an important role in the progression of cardiac dysfunction $[2,10]$.

To incorporate individualized properties of living cardiac tissue, in the presented study, we quantify cardiac mechanics by analyzing the actual movement of the heart. We utilize non-invasive magnetic resonance (MR) imaging measurements, and mathematically derive myocardial material properties for each subject. The FE calculation combines geometry and material properties derived in vivo, on an individual basis. Therefore, this technique provides realistic information to understand the human cardiac mechanism and may be applied to investigate wall stress changes for patients with heart disease.

\section{Methods}

\subsection{Imaging and model construction}

Tagged MR imaging was performed on two patients (S1 and S2) in the same age group (40's) with no history of cardiac disease. MR imaging measurements were obtained using a 1.5 Tesla scanner (Magnetom Avanto, Siemens Medical Solutions). MR images were acquired, gated to the electrocardiogram (ECG) in cross sectional slice format through the base, mid, and apical portion of the ventricles, as well as along two longitudinal cardiac axes. Each series of cine images comprised 18 phases corresponding to sequential stages of the cardiac cycle. $3 \mathrm{D}$ reconstruction of ventricles was based on images at relaxation state as it is defined by Tortora and Grabowski [11]. The heart rate and brachial artery pressure was recorded for calculation of myocardial material properties.

\subsection{Myocardial material properties}

In this section, we constructed a new formulation to drive the patient-specific myocardial material properties. In this derivation, only parameters that can be obtained from images or through non-invasive regular clinical setting were used. 
Therefore, it can be easily applied to medical image analysis with no additional procedures needed and give quantitative patient myocardial properties.

\subsubsection{Large elastic deformation material formulation}

The muscular fibers have a helical arrangement in a thick-walled cylinder representing the left ventricle (LV), shown in Figure 1. The orientation of the helical fibers has an angle $\gamma(r)$ with the Z-axis of the cylinder. Axial symmetry is assumed so that the variables are independent of $\phi$ in a cylindrical coordinate system. According to previous study by Chadwick [12] and Shoucri [13], the tension $T(r, z)$ generated by a muscular fiber during contraction will induce an active stress, $q_{i j}$, which can be expressed in cylindrical coordinates with components

$$
\begin{gathered}
q_{\phi z}=T(\sin \gamma)(\cos \gamma), \quad q_{r \phi}=q_{r z}=0 \\
q_{r r}=0, \quad q_{\phi \phi}=T \cos ^{2} \gamma, \quad q_{z z}=T \sin ^{2} \gamma
\end{gathered}
$$

Therefore, the total stress in the myocardium $\left(t_{i j}\right)$ can be expressed as

$$
t_{i j}=\sigma_{i j}-q_{i j}
$$

$\sigma_{i j}$ is the stress generated by the deformation of the passive medium of the myocardium and can be expressed by means of the constitutive relations of the medium. The myocardium can be considered as an incompressible isotropic passive medium surrounding the muscular fibers. In this case the strain energy function can be expressed as $W\left(I_{B}, I I_{B}\right)$ (eqn (3))

$$
W=C_{1}\left(I_{B}-3\right)+C_{2}\left(I I_{B}-3\right)
$$

where $C_{1}$ and $C_{2}$ are constants. $I_{B}$ and $I I_{B}$ are the first and second invariant of $\mathbf{B}$ (the left Cauchy-Green tensor), given in terms of the components of $\mathbf{B}$ as

$$
I_{B}=b_{i i} \quad I I_{B}=\frac{1}{2}\left(b_{i i} b_{j j}-b_{i j} b_{j i}\right)
$$

Neglecting the effect of residual stress, the cylinder will contract from the enddiastolic configuration described by $\left(r_{e d}, \phi_{e d}, z_{e d}\right)$ to a configuration $(r, \phi, z)$ according the relations

$$
r=\mu\left(r_{e d}\right) \quad \phi=\phi_{e d}+\lambda \psi z_{e d} \quad z=\lambda z_{e d}
$$




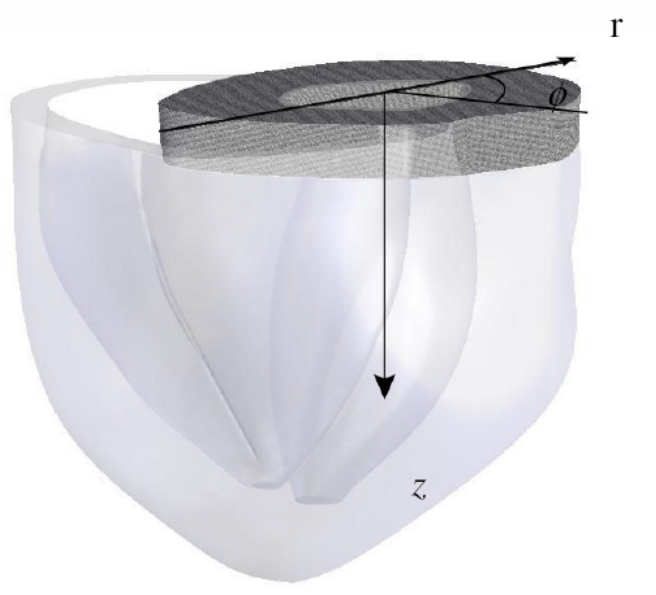

Figure 1: A human ventricular model. This biventricular configuration was constructed based on MR imaging data for human heart geometry (light gray). The dark gray region is the schematic representation to show the cylindrical coordinates $(r, \phi, z)$ for calculating the myocardial material properties.

$\lambda$ and $\psi$ are assumed constants. The local equilibrium of forces in the radial direction gives

$$
\frac{\partial t_{r r}}{\partial r}+\frac{t_{r r}-t_{\phi \phi}}{r}=0
$$

Substituting eqn (2) in eqn (6), we get

$$
\frac{\partial \sigma_{r r}}{\partial r}+\frac{\sigma_{r r}-\sigma_{\phi \phi}}{r}-D_{r}=0
$$

where $D_{r}=-q_{\phi \phi} / r$. To solve eqn (7), the stress component for $\sigma_{\phi \phi}$ (eqn (8)) and the boundary conditions of $\sigma_{r r}(a)=-P$ and $\sigma_{r r}(b) \approx 0$ ( $a=$ inner radius, $b=$ outer radius) was used [14]. Therefore, eqn (7) can be integrated and become eqn (9)

$$
\begin{gathered}
\sigma_{\phi \phi}=\sigma_{r r}+2 C_{1}\left(\mu^{2}+\lambda^{2} \psi^{2} r^{2}-\frac{1}{\lambda^{2} \mu^{2}}\right)+2 C_{2}\left(\lambda^{2} \mu^{2}-\frac{1}{\mu^{2}}\right) \\
\sigma_{r r}(r)=2\left(C_{1}+\lambda^{2} C_{2}\right) \int_{b}^{r}\left(\mu^{2}-\frac{1}{\lambda^{2} \mu^{2}}\right) \frac{d r}{r}+2 \lambda^{2} \psi^{2} C_{1} \int_{b}^{r} r d r+\int_{b}^{r} D_{r} d r
\end{gathered}
$$


Then we applied the mean value theorem and used the relation $\int_{a}^{b} D_{r} d r=\bar{D}_{r} h$ and $\mu=r / r_{e \mathrm{~d}}$ to obtain eqns (10) and (11)

$$
\begin{gathered}
\bar{D}_{r} h-P=2\left(C_{1}+\lambda^{2} C_{2}\right) \int_{a}^{b}\left(\frac{1}{\lambda^{2} \mu^{2}}-\mu^{2}\right) \frac{d r}{r}-2 \lambda^{2} \psi^{2} C_{1} \int_{a}^{b} r d r \\
\bar{D}_{r} h-P=\left(C_{1}+\lambda^{2} C_{2}\right)\left(\frac{1}{\lambda^{2}}\left[\frac{1}{\mu_{a}^{2}}-\frac{1}{\mu_{b}^{2}}\right]+\frac{2}{\lambda} \ln \frac{\mu_{b}}{\mu_{a}}\right)-\lambda^{2} \psi^{2} C_{1}\left(b^{2}-a^{2}\right)
\end{gathered}
$$

where $\mu_{a}=a / a_{e d}$, and $\mu_{b}=b / b_{e d}$

\subsubsection{Left ventricular chamber pressure}

To obtain the boundary conditions and solve eqn (11), we assumed that the peak left ventricular chamber pressure $L V_{\text {peak }}$ can be approximated by the systolic brachial artery pressure $P_{b}$

$$
L V_{\text {peak }}=P_{b}
$$

The left ventricular chamber pressure, $L V P$, can be expressed as eqn (13) for the duration from the beginning of ventricular systole to the end of isovolumetric relaxation [11]

$$
L V P(t)=L V_{\text {peak }} \sqrt{\frac{K^{2}-(2 t-K)^{2}}{K}}
$$

where $K$ is the time duration from the beginning of ventricular systole to the end of isovolumetric relaxation. Therefore, as long as we know the time at which $\mu$, $\lambda$ and $\psi$ were measured from we can calculate LVP and solve eqn (11). As mentioned in Section 2.1, the MR images used were ECG gated; therefore, $\mathrm{K}$ and $t$ were determined directly from the image itself.

Therefore, $P$ in eqn (11) can be calculated from eqn (14)

$$
P=L V P_{t 2}-L V P_{t 1}
$$

\subsection{The wall stress calculation}

The ventricular WS, $\{\sigma\}$, was calculated for each patient by solving eqns (15) and (16) [15] 


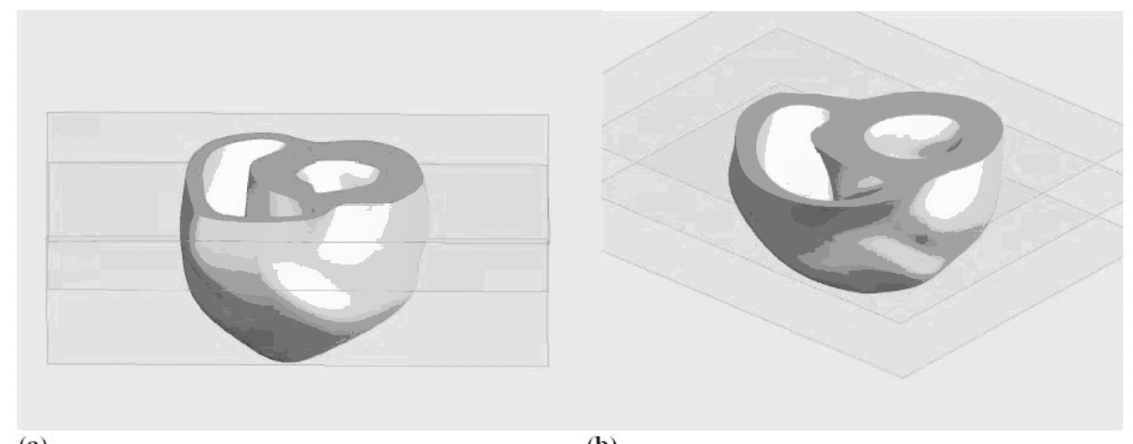

(a)

(b)

Figure 2: $\quad$ Gross models of ventricles for S1 (a) and S2 (b).

$$
\begin{gathered}
{[\mathbf{K}]\{\mathbf{D}\}=\{\mathbf{R}\}} \\
\{\boldsymbol{\sigma}\}=[\mathbf{E}][\mathbf{B}]\{\mathbf{D}\}
\end{gathered}
$$

where $\mathbf{K}$ is the stiffness matrix, $\mathbf{D}$ is the displacement vector, $\mathbf{R}$ is the load vector, $\mathbf{E}$ is the elasticity matrix, and $\mathbf{B}$ is the strain matrix. To achieve the goal of patient-specific modelling, the calculation was based on patients' ventricular geometry and heart motion. We used their MR images (section 2.1) to reconstruct the ventricular geometry and measurements of $a, b, \mu, \lambda, \psi, P_{b}$, and $K$ in order to calculate the material properties of the myocardium (section 2.2). Then the finite element method was applied to combine the geometry and material properties into one matrix, $\mathbf{K}$, to solve the wall stress equations (eqn (16)). We used parabolic tetrahedral elements with approximately $10^{4}$ elements for each biventricular model, and employed COSMOS DesignStar for the FE calculation.

\section{Results}

3D ventricular geometries were reconstructed for $\mathrm{S} 1$ and $\mathrm{S} 2$, shown in figure 2. Figure 2(a) is the $3 \mathrm{D}$ reconstruction for $\mathrm{S} 1$; figure 2(b) is for $\mathrm{S} 2$. Then the material properties were calculated using parameters measured from MR images. Table 1 showed the measurements made on the images. Parameters a1-a4 are the LV inner radii corresponding to image frame 1 , frame 2 , frame 3 , and frame 4.

The measurements for the outer radius are likewise labelled. The LVP values in Table 1 are the LVP given in the corresponding images frames. They were calculated using measurements from patient's brachial artery pressure, $\mathrm{K}$, and $\mathrm{t}$ values shown in Table 2 .

The material parameters (Table 3) were solved by eqns (11) and (14) using $\bar{D}_{r} h-P \approx 2 P$ for the normal physiological state of the heart. $C_{1}$ and $C_{2}$ are the 
parameters for the myocardial material in eqn (3) and were used in the $\mathrm{FE}$ calculation. The FE results for the end-systolic WS are shown in figure 3 using Von Mises stress (root-mean-square stress) definition. Results showed that at the end of the systole, ventricular outer walls had a wavy stress distribution. This was observed in both S1 and S2 (figure 3 left). We also found that in both cases, the inner WS was higher in the left ventricle compared with the WS in the right ventricle (figure 3 middle). The major difference in S1 and S2 was the WS pattern in the LV inner wall. S1 showed a more continuous area of high stress(red area), shown in figure 3 right-top. S2 showed a discrete high stress area (red area) shown in figure 3 right-bottom.

Table 1: Parameters to calculate myocardial material curves.

\begin{tabular}{|c|c|c|c|c|c|c|c|c|c|c|c|c|}
\hline & \multicolumn{1}{|c|}{ Inner radius $(\mathrm{mm})$} & \multicolumn{4}{c|}{ Outer radius $(\mathrm{mm})$} & \multicolumn{5}{c|}{$\mathrm{LVP}(\mathrm{mmHg})$} \\
\hline & $\mathrm{a} 1$ & $\mathrm{a} 2$ & $\mathrm{a} 3$ & $\mathrm{a} 4$ & $\mathrm{~b} 1$ & $\mathrm{~b} 2$ & $\mathrm{~b} 3$ & $\mathrm{~b} 4$ & $\mathrm{t} 1$ & $\mathrm{t} 2$ & $\mathrm{t} 3$ & $\mathrm{t} 4$ \\
\hline $\mathrm{S} 1$ & 15 & 14 & 13.5 & 11.5 & 32.5 & 32 & 30 & 28.5 & 8.0 & 94.5 & 125.0 & 141.8 \\
\hline $\mathrm{S} 2$ & 17.5 & 16.5 & 15.5 & 14.5 & 31 & 30.5 & 30 & 29 & 8.0 & 81.9 & 108.4 & 122.9 \\
\hline & Twist $(\psi), \mathrm{S} 1=0.07123 ; \mathrm{S} 2=0.0456$ \\
$\mathrm{Z}$ direction tension ratio $(\lambda), \mathrm{S} 1=0.938 ; \mathrm{S} 2=0.956$ \\
\hline
\end{tabular}

Table 2: $\quad$ Parameters to calculate LVP.

\begin{tabular}{|c|c|c|c|c|c|c|}
\hline & $\begin{array}{c}\text { Brachial artery pressure } \\
(\text { systolic/diastolic }) \\
(\mathrm{mmHg})\end{array}$ & $\begin{array}{c}\mathrm{K} \\
(\mathrm{msec})\end{array}$ & $\begin{array}{c}\mathrm{t} 1 \\
(\mathrm{msec})\end{array}$ & $\begin{array}{c}\mathrm{t} 2 \\
(\mathrm{msec})\end{array}$ & $\begin{array}{c}\mathrm{t} 3 \\
(\mathrm{msec})\end{array}$ & $\begin{array}{c}\mathrm{t} 4 \\
(\mathrm{msec})\end{array}$ \\
\hline $\mathrm{S} 1$ & $150 / 70$ & 517 & 57.5 & 115.0 & 172.5 & 230.0 \\
\hline $\mathrm{S} 2$ & $130 / 80$ & 566 & 62.9 & 125.8 & 188.7 & 251.6 \\
\hline
\end{tabular}

Table 3: Myocardial material properties.

\begin{tabular}{|l|l|c|}
\hline S1 & $C_{1}$ & -108.85 \\
\cline { 2 - 3 } & $C_{2}$ & -934.13 \\
\hline \multirow{3}{*}{ S2 } & $C_{1}$ & -82.25 \\
\cline { 2 - 3 } & $C_{2}$ & 349.56 \\
\hline
\end{tabular}

\section{Discussion}

This study suggests that in the living human heart the calculation of ventricular wall stresses is possible, based on analyzing tagged MR imaging data and some reasonable assumptions. 


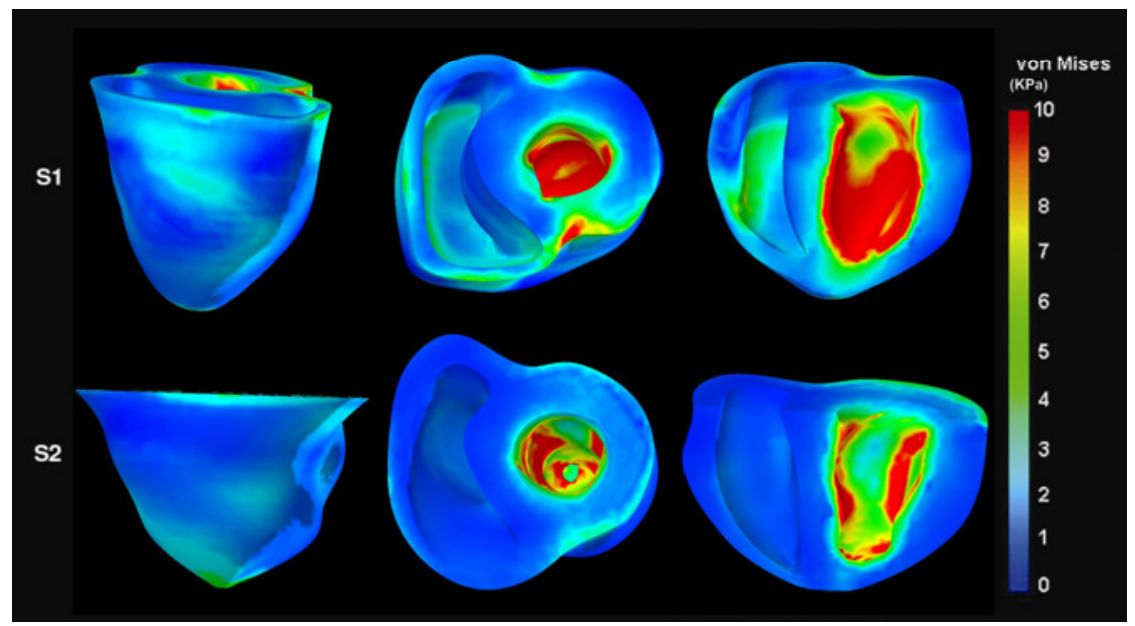

Figure 3: $\quad \mathrm{S} 1, \mathrm{~S} 2$ Von Mises stress distribution at the end of systole. The outer wall (left), two-chamber inner walls (middle), and LV inner wall (right) are shown. At the ventricular outer wall (left) a wavy stress distribution was observed (light blue). Two chamber views (middle) show both S1 and S2 had higher stress (red) in the left ventricle. The stress distribution in the LV inner wall showed that S1 (right-top) had a more continuous area of high stress (red); S2 (right-bottom) had a discrete high stress area (red).

In this paper, we derived patient's myocardial material properties based on dimensions of the heart, blood pressure and heart rate. This information is usually used in clinical assessments to evaluate patients' cardiac condition. The proposed method combines them through mathematical formulas; therefore, the calculated myocardial properties can realistically represent patient's myocardial material properties. In the results, we showed the myocardial properties for the two patients. Coefficients $C_{1}$ and $C_{2}$ were very different in these two cases although they both had no history of cardiac disease and were of similar age. We went back to the database and tried to understand the meaning of the results and found that of these two cases, one is male and one is female. Our hypothesis is that the variation in $C_{1}$ and $C_{2}$ may be the result of gender difference. Further confirmation of this hypothesis can be done with more patient data.

Combining the input of patient-specific ventricular geometry and material properties, the FE calculation showed that S1 and S2 had the same trend in WS distributions. Both showed higher inner wall stress than outer wall stress, and the WS was higher in the LV than the rest of the ventricles. This agrees with previous calculations by $\mathrm{Hu}$ et al [1]. However in Hu's study, they analyzed the WS distribution in human hearts with piece-wise linear material properties. The initial value for the elastic modulus estimation was obtained from a post-mortem canine heart study, which gave a higher elastic modulus, in other words the tissue was stiffer in their case. Therefore, in Hu's study, the WS was calculated 
to be ten times higher than found with our results. Since the method presented in this paper employed large elastic deformation theory and measurements based on patients' medical images, the calculated WS is more realistic and more applicable to understand human cardiac function.

Although we have not done a validation study for the presented calculation, the general agreement that we found among these two patients and a previous study [4] indicate that the results are valid and provide valuable information to understand individual differences in myocardial wall stress. The next step is to confirm the validity by comparing strain calculated from FE analysis with strain measured directly from MR imaging. In addition, since many cardiac dysfunctions involve not only myocardial deterioration but also changes in ventricular shapes, we would like to investigate the myocardial WS in those patients using this patient-specific approach. We would like to study how the WS varies among different cardiac disease group with a calculation that combines physiological data and geometry.

\section{Acknowledgements}

The authors are grateful to help from Howard Dinh, MD., and J Paul Finn, MD., for providing tagged MR images and to the UCLA Cardiovascular Magnetic Resonance Research Center, which generously shared technical knowledge in medical imaging when the project was developed.

\section{References}

[1] Hu, Z., Metaxas, D. \& Axel, L., In vivo strain and stress estimation of the heart left and right ventricles from MRI images. Med Image Anal, 7, pp. 435-444, 2003.

[2] Fonseca, C.G., Oxenham, H.C., Cowan, B.R., Occleshaw, C.J. \& Young, A.A., Aging alters patterns of regional nonuniformity in LV strain relaxation: a 3-D MR tissue tagging study. Am J Physiol Heart Circ Physiol, 285, pp. H621-630, 2003.

[3] Arts, T., Delhaas, T., Bovendeerd, P., Verbeek, X. \& Prinzen, F.W., Adaptation to mechanical load determines shape and properties of heart and circulation: the CircAdapt model. Am J Physiol Heart Circ Physiol, 288, pp. H1943-1954, 2005.

[4] Yin, F.C., Ventricular wall stress. Circulation Research, 49, pp. 829-842, 1981.

[5] Guccione, J.M., Walker, J.C., Beitler, J.R., Moonly, S.M., Zhang, P., Guttman, M.A., Ozturk, C., McVeigh, E.R., Wallace, A.W., Saloner, D.A. $\&$ Ratcliffe, M.B., The effect of anteroapical aneurysm plication on endsystolic three-dimensional strain in the sheep: a magnetic resonance imaging tagging study. J Thorac Cardiovasc Surg, 131, pp. 579-586 e573, 2006.

[6] Aikawa, Y., Rohde, L., Plehn, J., Greaves, S.C., Menapace, F., Arnold, M.O., Rouleau, J.L., Pfeffer, M.A., Lee, R.T. \& Solomon, S.D., Regional 
wall stress predicts ventricular remodeling after anteroseptal myocardial infarction in the Healing and Early Afterload Reducing Trial (HEART): an echocardiography-based structural analysis. Am Heart J, 141, pp. 234242, 2001.

[7] Moustakidis, P., Maniar, H.S., Cupps, B.P., Absi, T., Zheng, J., Guccione, J.M., Sundt, T.M. \& Pasque, M.K., Altered left ventricular geometry changes the border zone temporal distribution of stress in an experimental model of left ventricular aneurysm: a finite element model study. Circulation, 106, pp. I168-175, 2002.

[8] Walker, J.C., Ratcliffe, M.B., Zhang, P., Wallace, A.W., Fata, B., Hsu, E.W., Saloner, D. \& Guccione, J.M., MRI-based finite-element analysis of left ventricular aneurysm. American journal of physiology. Heart and circulatory physiology, 289, pp. H692-H700, 2005.

[9] Chien, A., Boyle, N. \& Montemagno, C., Nonuniform increases in regional wall stress encircle infarcted myocardium: a derivation using left ventricular simulation. Journal of Cardiac Failure, 10, pp. s32, 2004.

[10] Bree, D., Wollmuth, J.R., Cupps, B.P., Krock, M.D., Howells, A., Rogers, J., Moazami, N. \& Pasque, M.K., Low-dose dobutamine tissue-tagged magnetic resonance imaging with 3-dimensional strain analysis allows assessment of myocardial viability in patients with ischemic cardiomyopathy. Circulation, 114, pp. I33-36, 2006.

[11] Tortora, G.J. \& Grabowski, S.R., Principles of anatomy and physiology, Tenth Edition John Wiley \& Sons, Inc.: New York, pp. p.659-683, 2003.

[12] Chadwick, R.S., Mechanics of the left ventricle. Biophysical Journal, 39, pp. 279-288, 1982.

[13] Shoucri, R.M., Theoretical study of pressure-volume relation in left ventricle. American Journal of Physiology, 260, pp. H282-291, 1991.

[14] Green, A.E.a.A., J.E., Large Elastic Deformations and Nonlinear Continuum Mechanics Oxford University Press: London, pp. 42-45, 1960.

[15] Cook, R.D., Finite element modeling for stress analysis John Wiley \& Son, Inc.: New York, pp., 1995. 